\title{
Adaptive optics program update at TMT
}

\section{Boyer, B. Ellerbroek}

C. Boyer, B. Ellerbroek, "Adaptive optics program update at TMT," Proc. SPIE 9909, Adaptive Optics Systems V, 990908 (26 July 2016); doi: $10.1117 / 12.2232945$

SPIE Event: SPIE Astronomical Telescopes + Instrumentation, 2016, Edinburgh, United Kingdom 


\title{
Adaptive Optics Program Update at TMT
}

\author{
C. Boyer", B. Ellerbroek \\ TMT Observatory Corp., 100 W. Walnut St, Pasadena, CA, USA 91124;
}

\begin{abstract}
The TMT first light AO facility consists of the Narrow Field Infra-Red AO System (NFIRAOS), the associated Laser Guide Star Facility (LGSF) and the AO Executive Software (AOESW). Design, fabrication and prototyping activities of the TMT first light AO systems and their components have significantly ramped up in Canada, China, France, and in the US. NFIRAOS is an order $60 \times 60$ laser guide star (LGS) multi-conjugate AO (MCAO) system, which provides uniform, diffraction-limited performance in the $\mathrm{J}, \mathrm{H}$, and $\mathrm{K}$ bands over $34 \times 34$ arc sec fields with 50 per cent sky coverage at the galactic pole, as required to support the TMT science cases. NFIRAOS includes two deformable mirrors, six laser guide star wavefront sensors, one high order Pyramid WFS for natural guide star AO, and up to three low-order, IR, natural guide star on-instrument wavefront sensors (OIWFS) and four on-detector guide windows (ODGW) within each client instrument. The first light LGSF system includes six sodium lasers to generate the NFIRAOS laser guide stars.

In this paper, we will provide an update on the progress in designing, prototyping, fabricating and modeling the TMT first light AO systems and their AO components over the last two years. TMT is continuing with detailed AO modeling to support the design and development of the first light AO systems and components. Major modeling topics studied during the last two years include further studies in the area of pyramid wavefront sensing, high precision astrometry, PSF reconstruction for LGS MCAO, LGSF wavefront error budget and sophisticated low order mode temporal filtering.
\end{abstract}

Keywords: Adaptive optics program, extremely large telescopes

\section{INTRODUCTION}

The first light Adaptive Optics (AO) architecture for the $\mathrm{TMT}^{[1]}$ has been defined to provide near-diffraction-limited wavefront quality and high sky coverage in the near infra-red (IR) for the first TMT science instruments IRIS (InfraRed Imaging Spectrograph) and IRMS (InfraRed Multislit Spectrometer) ${ }^{[2],[3]}$. It is a Laser Guide Star (LGS) Multi Conjugate AO (MCAO) architecture consisting of (i) the Narrow Field IR AO System (NFIRAOS) ${ }^{[4]}$, which feeds up to three science instrument ports after sensing and correcting for wavefront aberrations introduced by the atmospheric turbulence and the telescope itself, (ii) the Laser Guide Star Facility (LGSF), which generates multiple LGS in the mesospheric sodium layer with the brightness, beam quality and asterism geometry required by both NFIRAOS and later the second generation of TMT AO systems, and (iii) the Adaptive Optics Sequencer of the AO Executive Software, which automatically coordinates the operations of the AO systems with the remainder of the observatory for safe and efficient observations.

Significant progress has been made in designing, modeling and prototyping these systems and the associated AO components over the last two years. The NFIRAOS team has continued to perform important tradeoffs and overall final system design, sub-contracted the final design of 12 major sub-systems with Canadian industry, and used their MCAO prototype bench to test and validate PSF reconstruction algorithms. The LGSF team has also conducted important tradeoffs, developed test setups, prototyped and benchmarked components and is developing the preliminary design of the LGSF. In house work is being performed to bring the AO Executive Software design to a preliminary design level. On the AO component front, design, prototyping and tests continue for the deformable mirrors, the wavefront sensor (WFS) detectors and associated cameras, the real time controller and the lasers. Extensive work is being performed to

* cboyer@tmt.org; phone: 1626395 1623;www.tmt.org

Adaptive Optics Systems V, edited by Enrico Marchetti, Laird M. Close, Jean-Pierre Véran, Proc. of SPIE Vol. 9909,990908 (c) 2016 SPIE $\cdot$ CCC code: 0277-786X/16/\$18 · doi: 10.1117/12.2232945 
update and finalize the design requirement documents and interface control documents of the AO systems, AO components and their corresponding sub-systems, and to establish the traceability of these requirements. Finally, several $\mathrm{AO}$ modeling and analysis activities have been conducted in the areas of pyramid wavefront sensing, high precision astrometry, PSF reconstruction for LGS MCAO, and detailing the LGS and NGS wavefront error budgets to further support the development of requirements for of TMT AO systems and components.

\section{TMT FIRST LIGHT AO REQUIREMENTS AND ARCHITECTURE REVIEW}

The TMT top-level AO science requirements are summarized in Table 1. They have remained very stable since the last SPIE Astronomical Telescopes and Instrumentation Conference in 2014, apart from a slight increase in the size of the corrected field of view to match anticipated changes to the IRIS imager.

Table 1: TMT top-level first light AO requirements

\begin{tabular}{|c|c|}
\hline Requirements & Implied AO design requirements \\
\hline High sky coverage ( $50 \%$ at the galactic pole) & $\begin{array}{ll}\text { - } & \text { Laser guide star (LGS) AO. } \\
\text { - } & \text { Natural guide stars (NGS) in NIR to sense tip/tilt/focus } \\
\text { - } & \text { Multi-conjugate AO with } 2 \text { deformable mirrors (DMs) to } \\
\text { sharpen guide star images over a large field of view. }\end{array}$ \\
\hline $\begin{array}{l}\text { Diffraction limited performance in J, H, and K } \\
\text { bands (187nm RMS on axis; } 208 \mathrm{~nm} \text { RMS on } \\
\text { 34"x34"; zenith with median seeing) }\end{array}$ & $\begin{array}{l}\text { Laser guide star (LGS) Multi-Conjugate AO: } \\
\text { - Multiple (6) LGS and tomographic reconstruction to defeat the } \\
\text { cone effect. } \\
\text { - Two DMs for wide-field wavefront correction } \\
\text { - High spatial }(60 \times 60) \text { and temporal }(800 \mathrm{~Hz}) \text { sampling to } \\
\text { minimize the wavefront errors due to DM fitting and servo lag. } \\
\text { - Bright lasers (20W or more) to minimize the wavefront sensor } \\
\text { noise error. }\end{array}$ \\
\hline $\begin{array}{l}\text { Astrometry ( } 50 \mu \text { arc sec over } 30 " \text { in } \mathrm{H} \text { band for } \\
\text { a } 100 \text { second exposure) } \\
\text { Photometry ( } 2 \% \text { over } 30 " \text { at } \lambda=1 \mu \mathrm{m} \text { for a } 10 \\
\text { minute exposure) }\end{array}$ & $\begin{array}{ll}\text { - } & \text { Multi-conjugate AO with PSF reconstruction. } \\
\text { - } & \text { Distortion-free optical system. }\end{array}$ \\
\hline $\begin{array}{l}\text { High optical throughput }(85 \% \text { over } 0.8-2.5 \mu \mathrm{m} \\
\text { with goal of } 90 \% \text { over } 0.6-2.5 \mu \mathrm{m}) \\
\text { Low background ( } 15 \% \text { of ambient sky and } \\
\text { telescope) }\end{array}$ & $\begin{array}{l}\text { Cooled }\left(-30^{\circ} \mathrm{C}\right) \mathrm{AO} \text { system with strong requirement to minimize } \\
\text { the number of optical surfaces. }\end{array}$ \\
\hline Output ports ( 3 ports, f/15 with a 2 ' FoV) & ulti-conjugate $\mathrm{AO}$ with 2 instruments at first light. \\
\hline
\end{tabular}

The first light AO architecture for TMT (Figure 1) consists of the following major systems:

- NFIRAOS, which is located on the TMT Nasmyth platform and relays light from the telescope to 3 science instrument ports after sensing and correcting for wavefront aberrations introduced by atmospheric turbulence and the observatory itself. NFIRAOS includes two DMs conjugated at $0 \mathrm{~km}(63 \times 63)$ and at $11.8 \mathrm{~km}(76 \times 76)$ with the DM conjugated to the ground mounted on a tip/tilt stage to reduce the number of optical surfaces. It also includes six $60 \times 60$ LGS WFS (one on-axis, and five in a pentagon with a radius of $35 \operatorname{arcsec}$ ), a $60 \times 60$ NGS WFS for operation without laser and operates at $800 \mathrm{~Hz}$. 
- The LGSF generates multiple LGS in the mesospheric sodium layer with the brightness, beam quality, and asterism geometry required by both the first light AO system (NFIRAOS) and later the second generation of TMT AO systems. It includes: i) the lasers, which are attached to the inside of the $-\mathrm{X}$ elevation journal facing the TMT primary mirror, ii) the beam transfer optics optical path, which transports up to 9 laser beams in a square pattern along the telescope elevation structure to the telescope top end, iii) the LGSF top end, which formats and launches the laser asterisms (up to 4 different asterisms) to the sky from the laser launch telescope, and iv) the laser safety system.

- The On-Instrument wavefront sensors (OIWFS) of the NFIRAOS instruments dedicated for tip/tilt/focus sensing in the near IR (IRIS provides three OIWFS and IRMS only one), and four On-Detector Guide Windows in IRIS serving as truth tip/tilt sensors.

- The Adaptive Optics Sequencer of the AO Executive Software, which automatically coordinates the operations of NFIRAOS, the OIWFS/ODGW and the LGSF with the remainder of the observatory for safe and efficient observations.

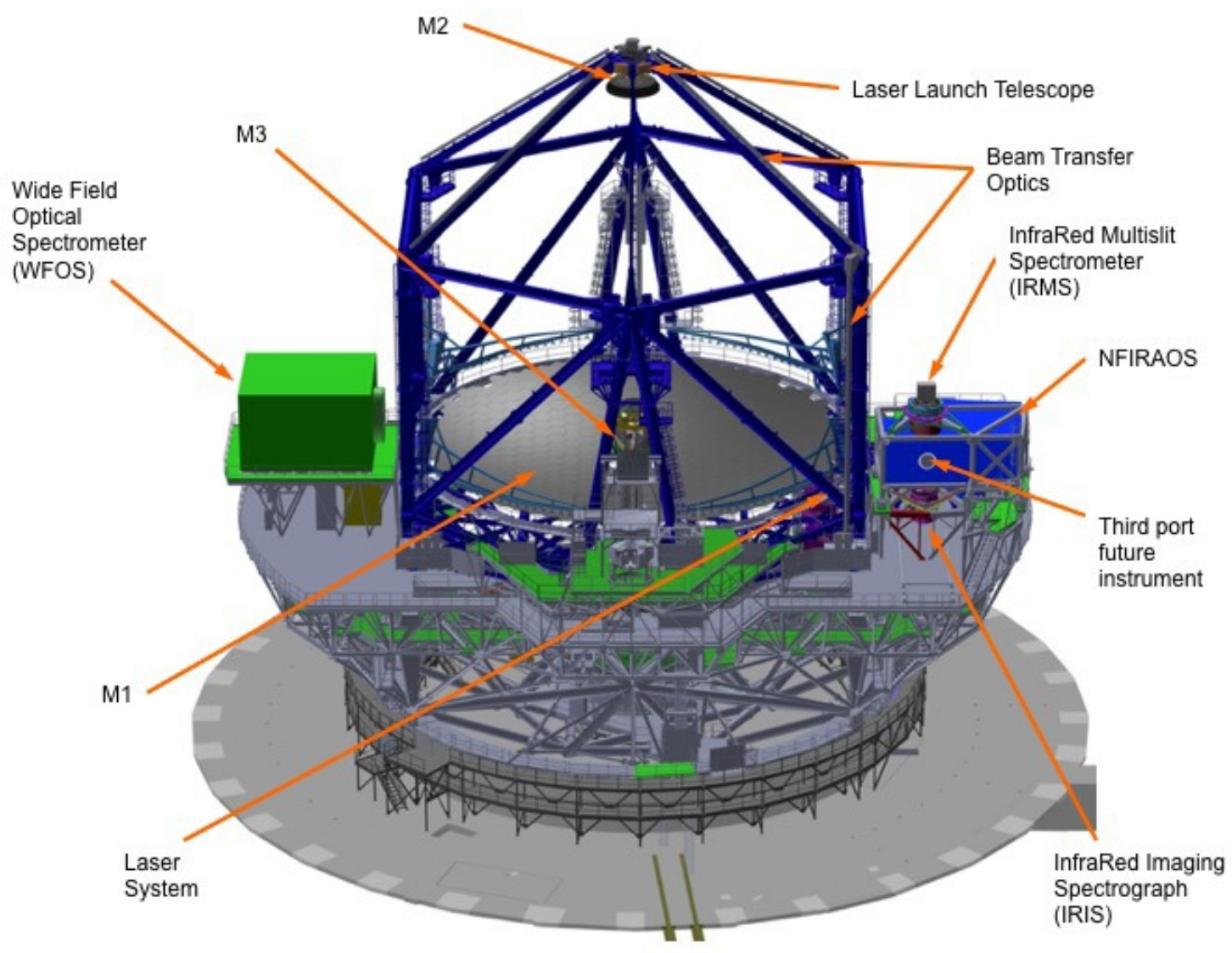

Figure 1: The TMT telescope with the first-light instruments and AO Systems (NFIRAOS and LGSF).

\section{FIRST LIGHT AO SYSTEM DESIGN PROGRESS}

\subsection{Narrow Field IR Adaptive Optics System (NFIRAOS)}

Since the SPIE Conference in 2014, the NFIRAOS team at NRC Herzberg in Victoria has been busy developing the final design. The updated NFIRAOS opto-mechanical design is given in Figure 2. 

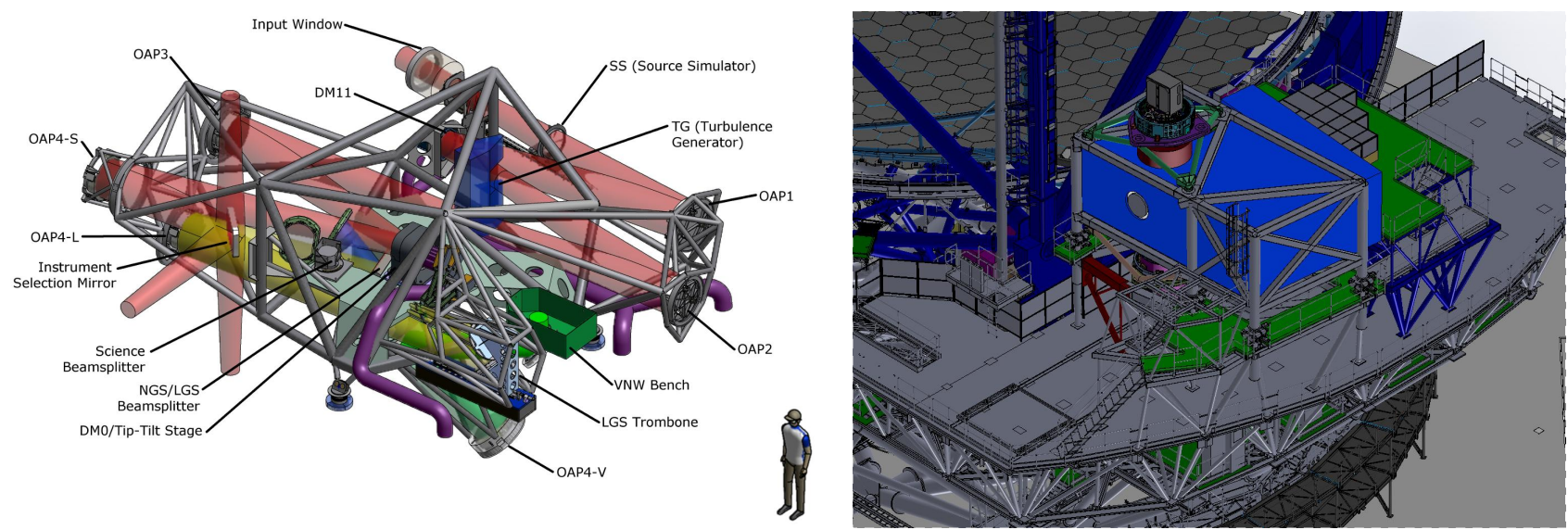

Figure 2: NFIRAOS opto-mechanical layout (left). Detail of NFIRAOS enclosure mounted on the Instrument Support Structure (right).

The main activities conducted by the NFIRAOS team are summarized below:

- Performed a NGS WFS trade study to select whether to use two Shack Hartmann WFSs or a Pyramid WFS for order NGS wavefront sensing (for NGS AO, potentially including high-contrast imaging) and Truth wavefront sensing to calibrate the LGS WFS for changes in the sodium layer profile (for LGS AO only) ${ }^{[6,[7]}$.

$\circ$ The opto-mechanical design of the Pyramid WFS is simpler than the Shack Hartmann WFS design. It has fewer optics and mechanisms as shown in Figure 3. The complexity of using a Pyramid WFS is out-weighted by the fact that only one sensor is needed instead of two in the Shack Hartmann WFS design.

- For NGS AO observations, the PWFS is less sensitive to noise and to aliasing, leading to improved performance. As described in Figure 3, the PWFS has a limiting magnitude 1.3 higher than the Shack Hartmann WFS for a 50\% Strehl ratio in H band, resulting in roughly a three-fold increase in sky coverage and exposure time saving of more than 30\%. For high-contrast imaging, the Pyramid WFS provides a significantly lower level of "pinned" speckles and corresponding improvements in the achievable contrast ratios..

- In LGS AO observations, the Pyramid WFS permits higher spatial and temporal sampling and enables more accurate real-time calibration of the LGS WFS reference vectors..

- Finally, the impacts on other AO systems, such that the NFIRAOS Real Time Controller, are small.

- The conclusion of the study was to use a Pyramid WFS to improve the performance of the NGS mode while keeping the design impact minimum.

- Developed the final design of NFIRAOS ${ }^{[5],[8],[9],[10],[11]}$. The overall system design, system engineering, and software and electronics design efforts are performed at NRC, while the final design of the main subsystems and opto-mechanical components is sub-contracted to Canadian industry. Eight sub-contracts have already been placed, and several more are expected to be placed by end of 2016. The Instrument Selection Mirror and Beam Splitter designs are now complete. Design work for the off-axis parabolas, source simulator, turbulence generator, instrument support tower and internal framework, optical enclosure and the diagnostic system (including a high resolution wavefront sensor, a diffraction limited camera and an acquisition camera) is on going.

- Additionally, extensive work has been done to develop the interfaces with other observatory sub-systems, particularly with the telescope structure to optimize the resonant frequencies of NFIRAOS under different seismic conditions.

- Used the MCAO test bench for testing various algorithms for wavefront sensing, wavefront reconstruction, non-common path aberration calibration and PSF reconstruction ${ }^{[12],[13]}$. 

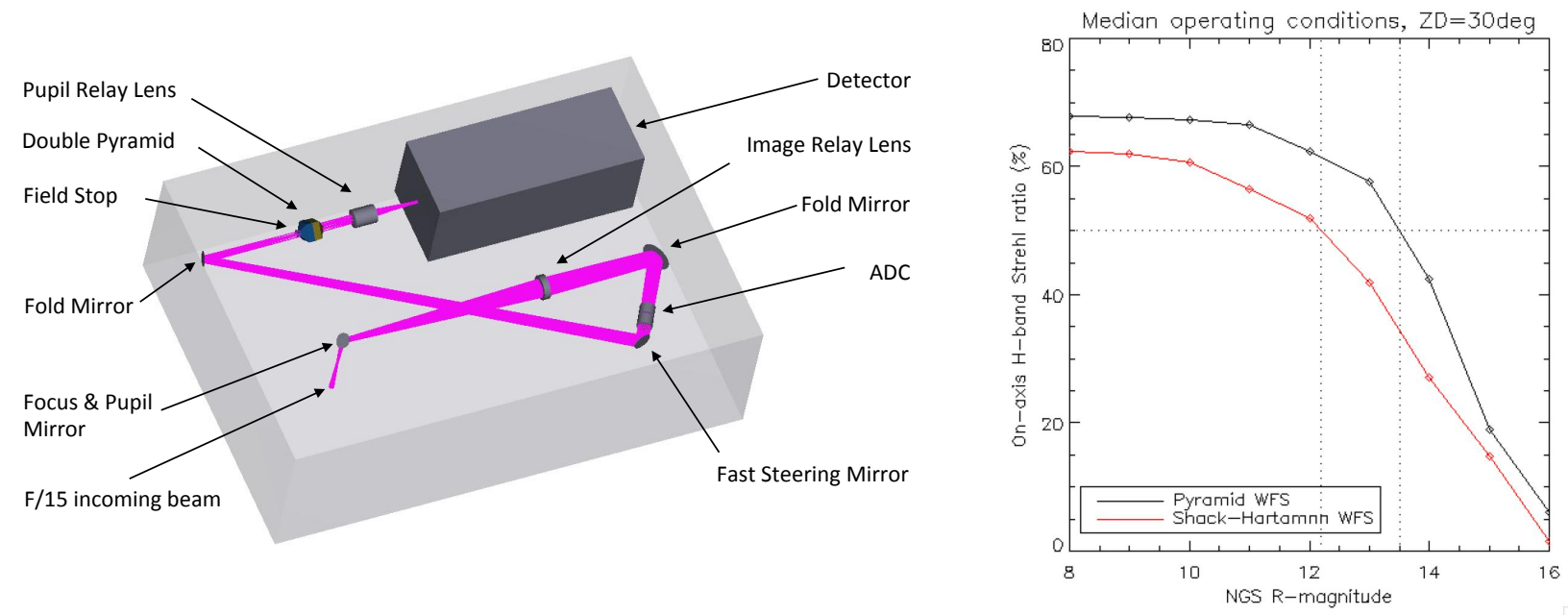

Figure 3: Design of the visible natural guide star bench including the pyramid WFS (left). Comparison of the H-band Strehl as a function of guide star magnitude (showing a gain of 1.3 magnitudes for the PWFS at $50 \%$ Strehl) (right).
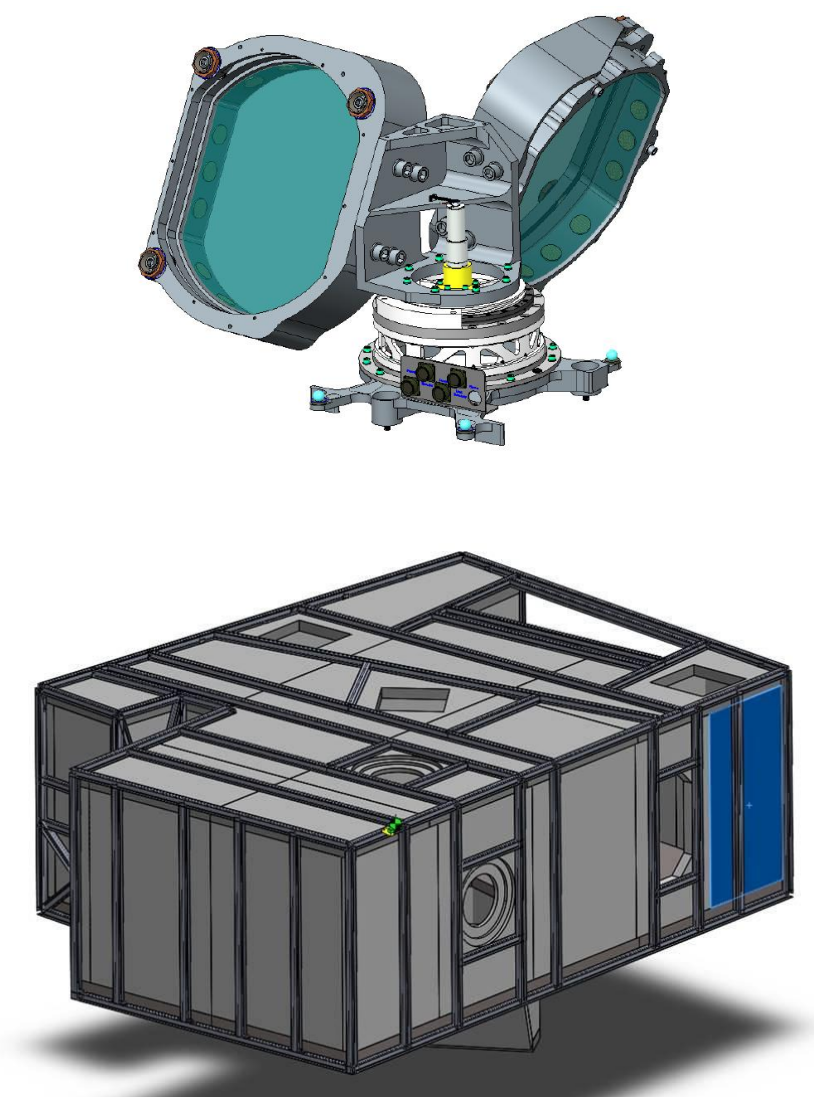
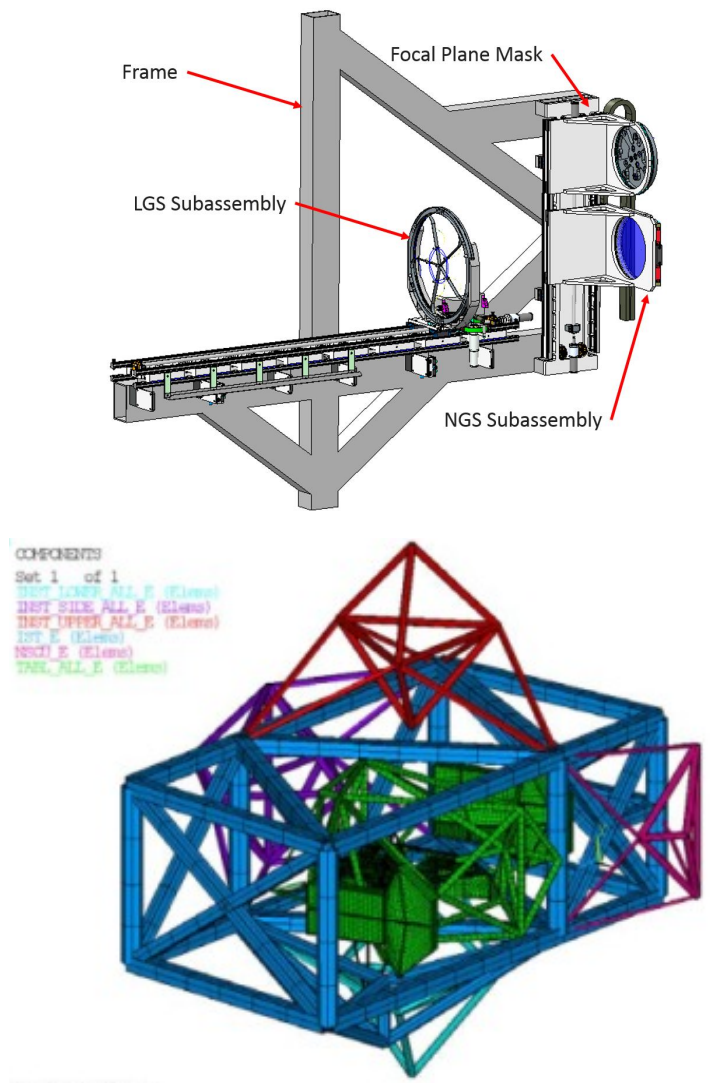

Figure 4: Final design of the NFIRAOS Beam Splitter (top left). Final design of the Source Simulator System including the LGS sources on a stage, the NGS sources and the focal plane mask (top right). Final design of the optical enclosure (bottom left). Full finite element model of NFIRAOS instrument support tower and internal frame structure (bottom right).

\subsection{Laser Guide Star Facility (LGSF)}

The Institute of Optics and Electronics (IOE) team in Chengdu, China is actively developing the LGSF preliminary design with the support of the TMT AO team. 

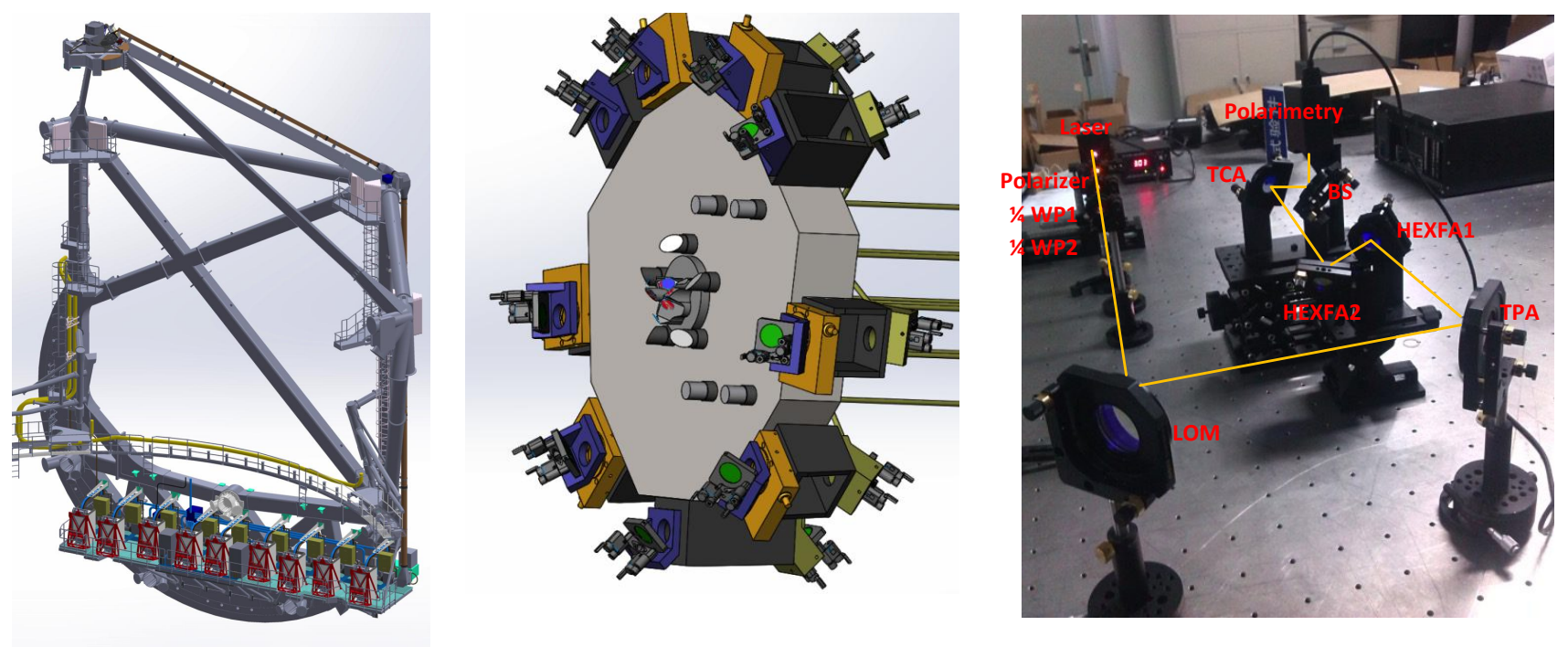

Figure 5: Detailed interface of the LGSF with the telescope structure (left). Details of the asterism generator design (middle). Polarization test bench (right)
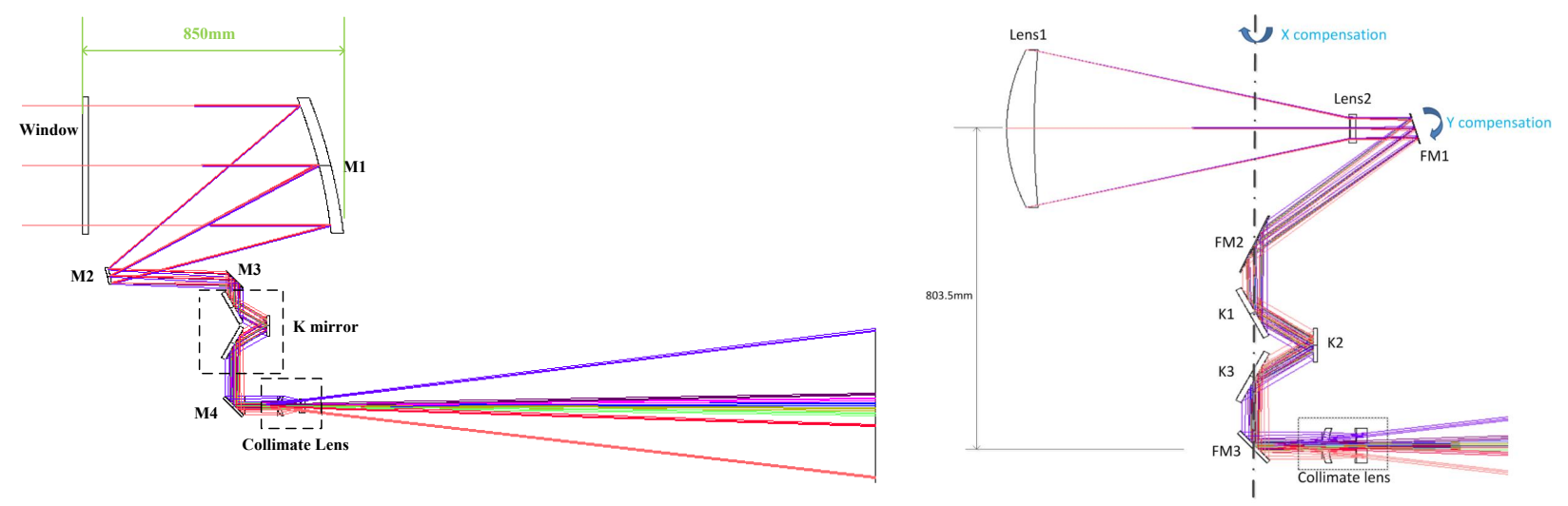

Figure 6: Laser Launch Telescope reflective off axis design (left). Laser Launch Telescope refractive design (right).

The main design activities performed by the IOE team are summarized below:

- Developed the detailed interface to the telescope structure. The LGSF system extends from the laser platform to the telescope top end, and has many complex structural, mechanical, access and handling, services and utilities, and safety interfaces to the telescope structure.

- Performed extensive optical modeling of the entire system to develop a detailed beam displacement budget at each node of the optical path, and evaluate the effect of optical figure and alignment errors on beam quality ${ }^{[16]}$.

- Conducted several tradeoffs for the Laser Launch Telescope (LLT) optical design (off axis reflective design versus refractive design), and whether to use individual beam control versus common control at the different nodes of the optical path for flexure correction.

- The original design for the Laser Launch Telescope was a reflective off axis beam expander as shown in Figure 6. Compensation of the telescope top end flexure was achieved by tilting the entire launch telescope around the $\mathrm{x}$-axis, and by pivoting the Laser Launch Telescope secondary mirror about the zero coma point in y. This design had excellent optical performance, but very tight tolerances to maintain a fixed separation between the primary and secondary mirrors under different gravity orientations and operating temperatures. 
- A new refractive design has been proposed as shown in Figure 6. As for the reflective design, top-end flexure compensation is achieved by tilting the entire launch telescope around the $\mathrm{x}$-axis, and by pivoting the first fold mirror in y. This design has very good optical performance, acceptable performance when compensating flexure around the y-axis, and significantly looser mechanical tolerances. Furthermore, the first fold mirror is ideal for fast tip-tilt correction of telescope wind-shake. The mechanical design of the refractive launch telescope is in progress, with fully passive control of temperature effects as a goal.

- The refractive design was selected to relax the mechanical tolerances while maintaining the performance of the LGSF system

- Developed prototyping and test setups for the uplink fast tip/tilt correction and beam polarization control (Two quarter wave plates per beam are necessary to ensure circularly polarized output).

- Developed the preliminary design of the different opto-mechanical components, including the laser benches, the common mounting structure of the laser heads and laser benches, the optical path components, and the top end sub-systems such as the diagnostic bench, the asterism generator, and the laser launch telescope... The preliminary design of the electronics, software and safety sub-systems is also progressing.

\subsection{Adaptive Optics Executive Software}

The AO Executive Software is composed of three sub-systems: i) the AO Sequencer, which coordinates the actions of the AO systems, ii) the Reconstructor Parameter Generator (RPG), which computes the AO parameters needed by the NFIRAOS Real Time Controller, and, iii) the Point Spread Function (PSF) Reconstructor, which post-processes the AOcorrected PSF from the NFIRAOS WFS and DM telemetry data.

A fully automated AO Sequencer is required due to the complexity of the interactions between AO systems and the TMT requirement for high observing efficiency. This software system will be central to performing AO-assisted observations. It will perform the control and monitoring of the NFIRAOS sub-systems, LGSF sub-systems, On-Instrument WFSs and On Detector Guide Windows of the NFIRAOS instruments. The AO Sequencer components will be dynamically created and composed to execute the startup, test, calibration, observation and shutdown sequences. The observation sequences also include sub-sequences for acquisition, nodding and dithering, non-sideral object tracking, calibration and recovery after a laser safety event or $\mathrm{AO} /$ telescope fault.

In house work to further update these sequences, formulate algorithms for the RPG, and complete the preliminary design of the AO Sequencer and RPG sub-systems is progressing and will be completed by end of 2016. The work to define the PSF Reconstruction algorithms is ongoing, with further algorithms tests planned at NRC with the MCAO bench and later with GEMS at Gemini South.

\section{FIRST LIGHT AO COMPONENT DEVELOPMENT}

\subsection{Deformable Mirrors}

The requirements for the NFIRAOS DMs includes two mirrors of order $63 \times 63$ and $76 \times 76$ with a $5 \mathrm{~mm}$ inter-actuator spacing, $10 \mu \mathrm{m}$ of stroke after flattening, $15 \%$ hysteresis, less than $2 \%$ per time decade creep and an operating temperature of $-30^{\circ} \mathrm{C}$.

During the last two years, TMT, ESO and CILAS have co-funded a new round of development to improve the manufacturing process of the CILAS actuators and their reliability. In parallel, TMT has contracted AOA Xinetics to develop an actuator meeting the TMT stroke and hysteresis requirements at $-30^{\circ} \mathrm{C}$. Both studies have been successful, and TMT has now launched two contracts with AOA Xinetics and CILAS ${ }^{[17]}$ to develop the final designs of the NFIRAOS DMs, and to fabricate new prototypes to be delivered to HIA for testing during the summer of 2017. Both contracts are progressing well at this point.

\subsection{High-order LGS and NGS Wavefront Sensing}

For the six NFIRAOS 60x60 LGS WFS, TMT will use the polar coordinate CCDs designed specifically for elongated laser guide star images. The polar coordinate $\mathrm{CCD}$ reduces the pixel count and total readout rate by roughly a factor of three in comparison to a standard CCD with a conventional rectangular geometry. The requirements for such a detector 
for TMT include sub-aperture sizes varying from 6x6 pixels at the center of the array to $6 \times 15$ pixels at the edge, a quantum efficiency of $90 \%$, and $\sim 3$ electrons read noise at a frame rate of $800 \mathrm{~Hz}$.

A one-quadrant prototype of the TMT polar coordinate CCD was designed and fabricated in a wafer run at MIT/LL funded by the TMT, Keck and USAF Research Laboratory. Tests of these prototype devices demonstrated fully functional outputs, good yield, uniform CTE, $85 \%$ QE, read noise level of 2.7 to 3.7 electrons at $3.5 \mathrm{MHz}$ (vs. a 3 electron requirement) and acceptable dark current at $800 \mathrm{~Hz}^{[18]}$.

The design of the full-scale detector has been completed by MIT/LL based on the successful prototype quadrant design. The quadrant design was mirrored twice to fill a $360 \mathrm{arc}$, and a third metal layer was added to avoid gaps between the quadrants. A first lot of sixteen, $200 \mathrm{~mm}$ wafers of $14 \mathrm{CCDs}$ each is being fabricated, with the first eight wafers fully processed through front illumination and now being tested. First test results of 10 science grade CCDs show good responsivity, good charge transfer efficiency (CTE), low dark current, and good cosmetics ${ }^{[20]}$. Further tests are ongoing to improve the yield for the remaining wafers.
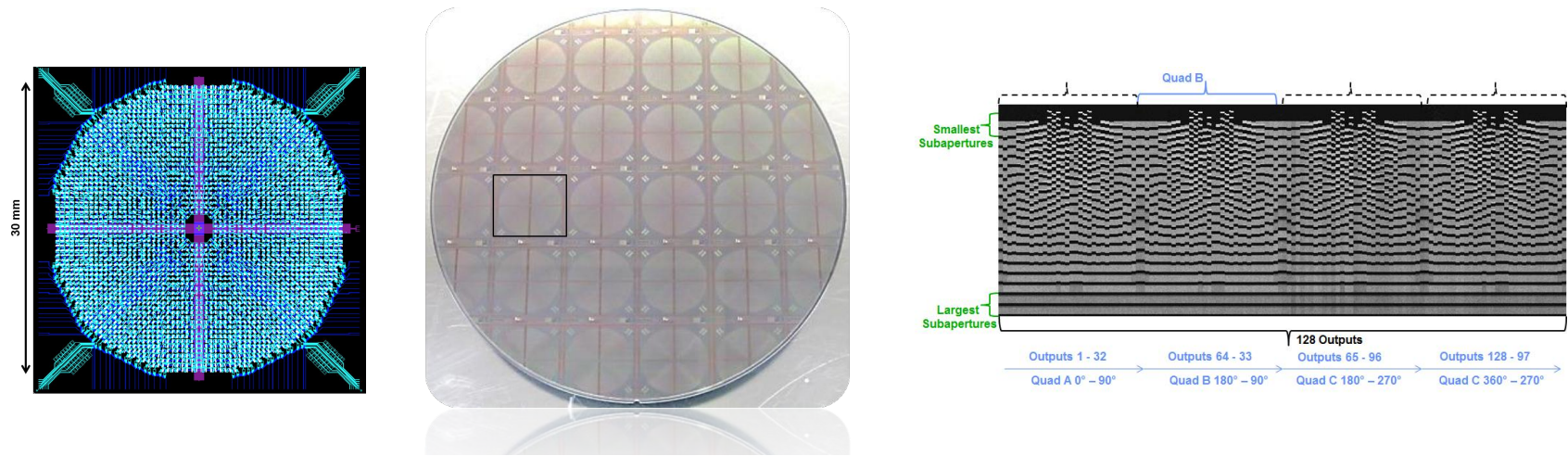

Figure 7: CAD image showing the layout of the polar coordinate device and the 3 metal layer design (left). One finished front illumination wafer (middle). Initial screening image (room temperature dark frame) showing responses of 128 outputs for one detector (right).

For the NFIRAOS NGS Pyramid WFS, TMT will use the 256x256 CCD array developed by MIT/LL as part of the polar coordinate prototype detector effort. This CCD has been successfully tested and achieves the requirements of $80 \%$ quantum efficiency and $\sim 1$ electron read noise at a $10 \mathrm{~Hz}-800 \mathrm{~Hz}$ frame rate ${ }^{[19]}$. Two science grade devices will be packaged for TMT using the same detector package developed for the polar coordinate detector. The LGS and NGS WFS cameras for NFIRAOS will also be based on a common design.

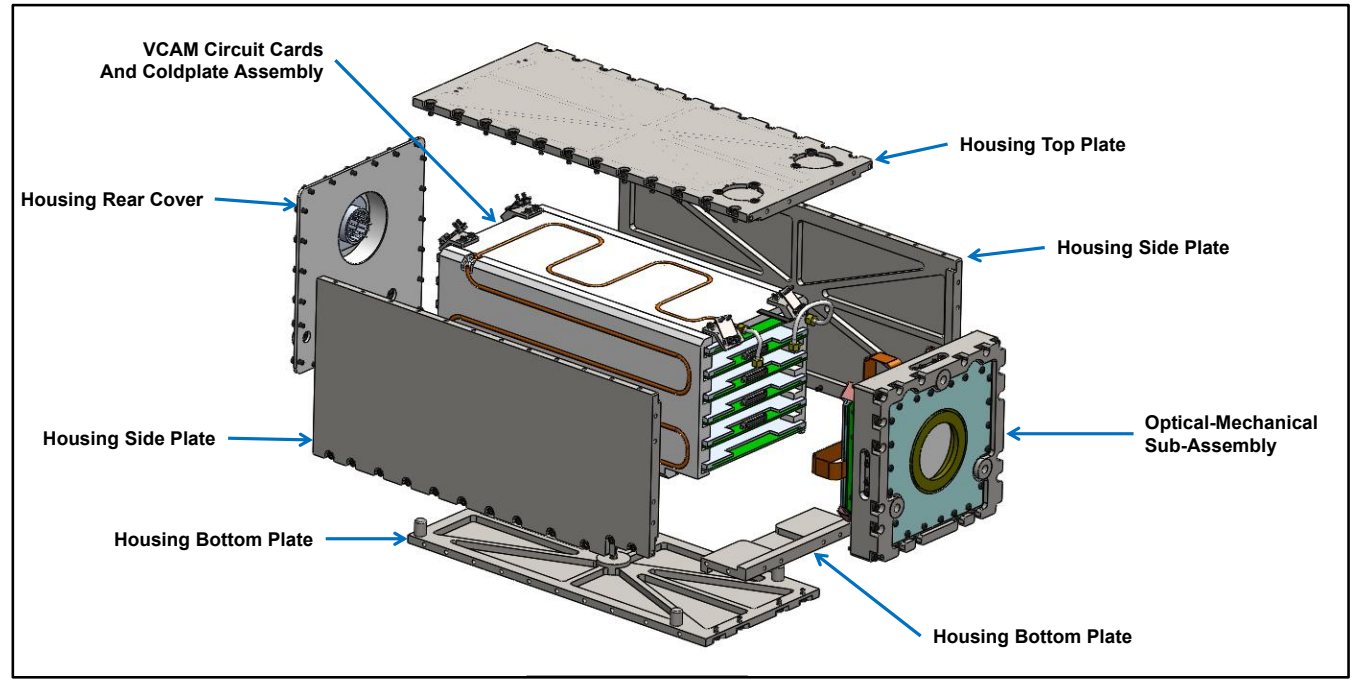

Figure 8: CAD image of the NFIRAOS LGS and NGS visible WFS cameras, including the focal plane readout electronics. 
The preliminary design of the NFIRAOS LGS and NGS WFS cameras and readout electronics has been contracted to Astronomical Research Cameras (ARC) in San Diego, with Quartus Engineering as a sub-contractor for the optomechanical design. The main performance requirements of the readout electronics and camera are summarized here:

- Fast pixel data rates for the readout electronics: 128 parallel outputs at 3.5 million pixels per second per output, with a total of $\sim 205,000$ pixels read out in $500 \mu \mathrm{sec}$ for the polar coordinate detector.

- Low noise for the readout electronics $(<3$ electrons for the polar coordinate detector and $<1$ electron for the NGS detector), requiring the electronics to be packaged close to the detector to avoid long and large interconnections.

- Small volume constraints and less than $0.5 \mathrm{~W}$ heat dissipation per LGS and NGS camera, as they are located within the $-30^{\circ} \mathrm{C}$ NFIRAOS cold enclosure.

The ARC/Quartus team has successfully completed the preliminary design of the cameras and readout electronics, and is now developing a 32 channel sub-scale readout electronics prototype. The plan is to test the prototype readout electronics with engineering grade polar coordinate detectors and the NGS WFS 256x256 detector in early 2017.

\subsection{Low-order On-Instrument NGS Wavefront Sensing}

TMT presently intends to use Teledyne Hawaii-2RG detectors with $1 \mathrm{Kx} 1 \mathrm{~K}$ science grade sub-arrays for the low-order On-Instrument WFS detectors in the NFIRAOS instruments. These detectors provide a large enough field of view for initial acquisition, very good quantum efficiency in the $\mathrm{J}, \mathrm{H}$ and $\mathrm{K}$ bands, and low read noise for small sub-arrays at frame rates of $10-400 \mathrm{~Hz}$. An alternative detector under consideration is the Selex APD. This detector appears to provide superior read noise performance on larger sub-arrays at the required frame rate, although the current device with $256 \mathrm{x}$ 320 pixels is still too small for initial guide star acquisition.

The use of on-instrument wavefront sensors has now been augmented with the definition of an on-detector guide window capability for the IRIS imager. Up to four guide windows can be used for either fast tip/tilt sensing or lowerbandwidth "truth" sensing of slowly varying alignment errors, depending upon the brightness of the guide stars. The use of the OIWFS and the ODGW will be fully flexible and interchangeable to the NFIRAOS RTC, apart from the requirement for at least one OIWFS for tip/tilt/focus sensing.

\subsection{Real Time Controller}

The requirements for the NFIRAOS Real Time Controller include real time pixel processing for the high-order LGS and low-order On-Instrument NGS wavefront sensors, tomographic wavefront reconstruction and calculation of the wavefront corrector actuator commands (requiring to solve a $35 \mathrm{k}$ x $8 \mathrm{k}$ control problem at $800 \mathrm{~Hz}$ ), and real-time optimization of the algorithms for these processes as atmospheric and observing conditions change. The RTC also acquires wavefront corrector and wavefront sensor telemetry data in order to estimate the science Point Spread Function (PSF) for image post-processing.

The NRC-Herzberg team has successfully completed the preliminary design of the NFIRAOS Real Time Controller. The system architecture is based upon commercial server hardware with a classical Matrix Vector Multiply (MVM) control algorithm. Intensive benchmarking have been conducted to support this architecture and have demonstrated that the performance requirements can be met by using a moderate number of existing off-the-shelf commercial boards, which in addition enable the use of a significantly simpler RTC algorithm (MVM) ${ }^{[14],[15]}$.

The RTC team has now launched the final design. The team will continue to survey the processor technology and in particular will check the performance of the next generation Intel Xeon Phi processor before selecting the final hardware architecture. 


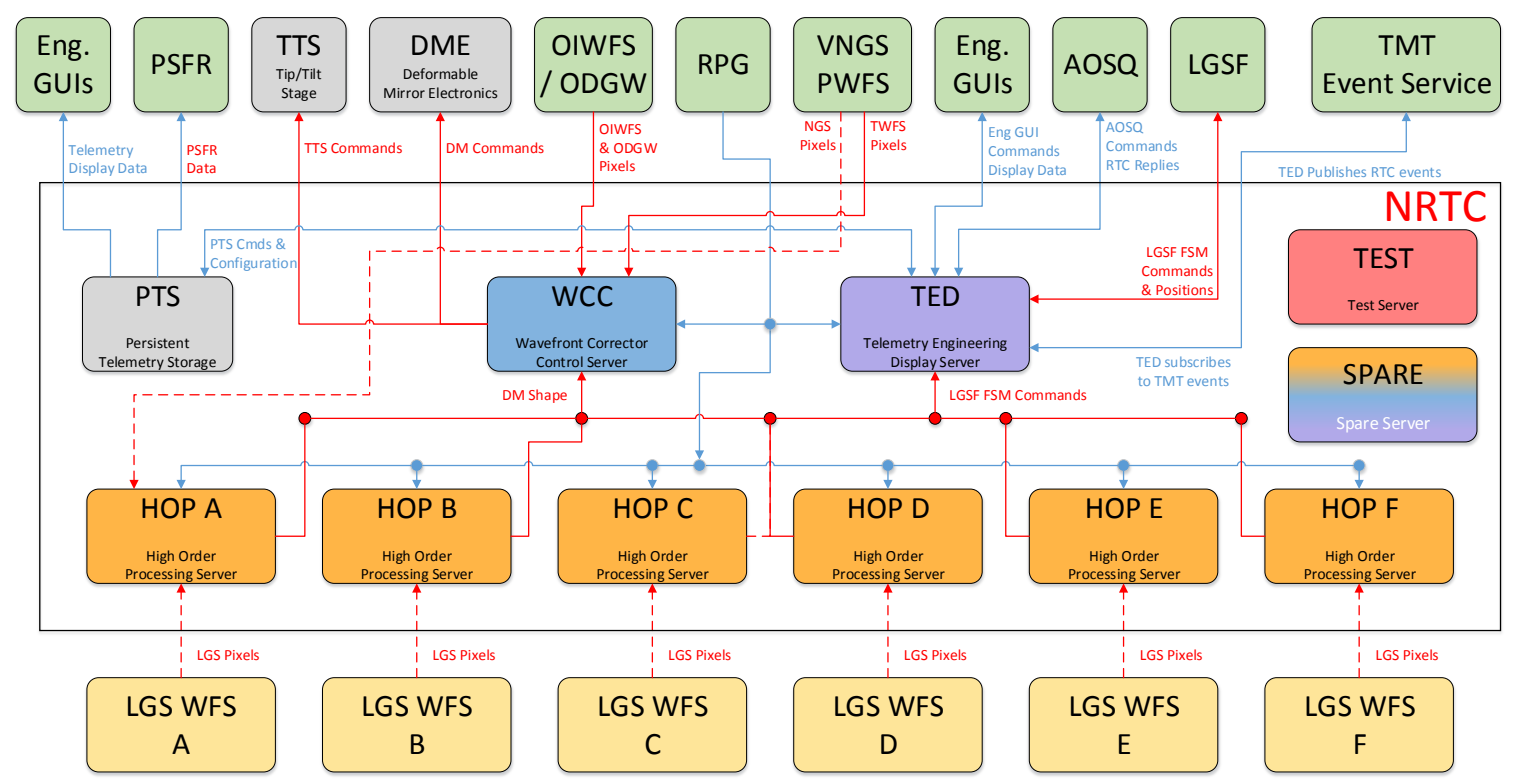

Figure 9: The RTC includes a total of ten servers with one server per LGS WFS, one server dedicated to wavefront corrector control, one server dedicated to telemetry engineering display, a test server and a spare server. Each server currently includes four high end Intel Xeon CPUs, although the Xeon Phi processor remains under consideration. The connection between the servers, to and from the AO components and other systems is performed through a 10/40Gb Ethernet switch with 32 ports.

\subsection{Lasers}

The TMT first light Laser Guide Star Facility will utilize six $25 \mathrm{~W}$ sodium guide star lasers $\left(20 \mathrm{~W}\right.$ with the $\mathrm{D}_{2} \mathrm{a} / \mathrm{D}_{2} \mathrm{~b}$ repumping option) with high beam quality, high coupling efficiency, high reliability, and a design compatible with a variable gravity vector orientation and the harsh environment of an astronomical observatory TMT is considering two candidate laser devices :

- The sodium laser developed by Toptica/MPBC for ESO and Keck. The Toptica laser is a Raman Fiber laser and produces the $589 \mathrm{~nm}$ beam using second harmonic generation (SHG) conversion of a $1178 \mathrm{~nm}$ laser ${ }^{[21]}$. The Toptica laser meets all the TMT performance and engineering requirements and has been successfully commissioned at the ESO VLT with the 4LGSF system, and at Keck with the Keck II NGL system ${ }^{[22],[23]}$.

- A second candidate is a sodium laser developed by the Technical Institute of Physics and Chemistry (TIPC) in Beijing, China. The laser design is based upon Sum Frequency Generation (SFG) using 1319nm and 1064nm $\mathrm{Nd}$ :YAG solid state lasers, and has a pulsed format with varying pulse length and pulse repetition frequency. The laser development is still at early age. Several TIPC laser prototypes have been built and tested in the lab and on the sky in China and in Canada between 2010 and 2016. The latest prototype meets most of the TMT performance requirements, with more than $25 \mathrm{~W}$ output power at a $800 \mathrm{~Hz}$ pulse repetition frequency. However, the prototype does not meet the beam quality requirement, and the results for coupling efficiency have only been demonstrated with spot sizes of at least 4.9 arcsec. Although significant progress has been achieved, the TIPC laser still has major challenging engineering requirements to demonstrate operation in a variable gravity orientation, reliability, cooling requirements, vibration....

TMT is now developing a cooling system concept for up to eight Toptica lasers. The cooling system will be mounted on the laser platform and will include a heat exchanger and a heater, which will be used to warm the laser electronics during the day and maintain the proper operating temperature during the night using the waste heat generated by the lasers while they are lasing. 

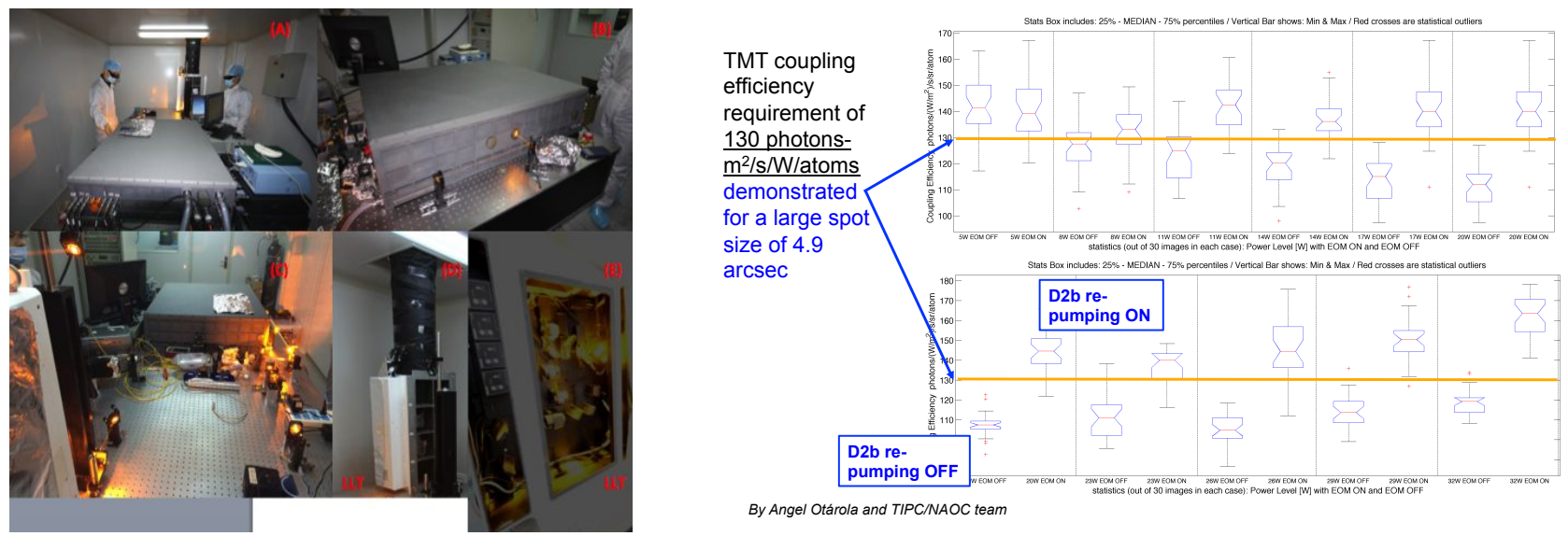

Figure 10: TIPC third prototype laser tested at Xinlong Observatory in China in 2014. Left: on-sky test setup. Right: Laser coupling efficiency as a function of power output demonstrated for a spot size of $4.9 \mathrm{arcsec}$ (Top plot: power output varying from $5 \mathrm{~W}$ to $20 \mathrm{~W}$. Lower plot: power output varying from $20 \mathrm{~W}$ to $32 \mathrm{~W}$. For each power output, the coupling efficiency has been estimated with the $\mathrm{D}_{2} \mathrm{~b}$ re-pumping option $\mathrm{ON}$ and $\mathrm{OFF}$ ).

\section{AO SYSTEM ENGINEERING, MODELING AND PERFORMANCE ANALYSIS}

Several AO modeling and analysis activities have been conducted at TMT, in particular in the areas of AO requirements definition, wavefront error budget development, pyramid WFS performance modeling for the NGS AO mode and truth wavefront sensing, and tip/tilt/focus sensing during guide star acquisition ${ }^{[25]}$. PSF Reconstruction modeling and experimental analysis has been started again using the NRC-Herzberg MCAO test bench with the goal to confirm the algorithms, and later test them with GeMS at Gemini South.

\subsection{AO System Engineering}

Extensive work to define the AO requirements and their traceability to/from requirements at higher or lower levels is underway at the TMT project office and the partner institutions. Similar attention is being directed to the interface control documents. The total number of requirements for the NFIRAOS, LGSF, and AOESW systems and the AO components sums up to about 1200 requirements, not including the lower level requirements for the sub-systems of NFIRAOS and the LGSF. There are also many interface requirements needing to be negotiated with other observatory or AO systems, split between a total of 41 interface control documents. More than half of the requirement documents are under change control. More than two thirds of the interface control documents have been written and are at different stage of maturity.

\subsection{AO Budget Updates}

The NFIRAOS wavefront error budget has been updated for the LGS MCAO operating mode to distinguish between requirements and current best estimates $(\mathrm{CBE})$, to incorporate design changes and new analysis results, and to better identify traceability to/from other requirements. In particular, the LGS MCAO current best estimate has been updated i) to account for the new 34"x34" IRIS imager FoV, ii) to further detail the NFIRAOS error terms (such as optical alignment and polishing terms), iii) to include the IRIS wavefront error budget, iv) to include the telescope mechanical vibration budget, v) to include the LGSF contribution to the LGS MCAO wavefront error budget and applicable error terms from the AO components, such as the DM dynamic effects. The top-level budget is given in Table 2 and the breakdown for the $\mathrm{AO}$ architecture term is provided in Table 3.

Similar work is ongoing to complete the wavefront error budget for the NGS AO operating mode and the astrometry and photometry budget ${ }^{[24]}$. 
Table 2: Updated top-level LGS MCAO performance estimates versus current top-level requirements.

\begin{tabular}{|c|c|c|c|c|}
\hline Field of View & \multicolumn{2}{|c|}{ On-Axis } & \multicolumn{2}{|c|}{ 34" x 34" FoV Average } \\
\hline Requirement, RMS nm & \multicolumn{2}{|c|}{187} & \multicolumn{2}{|c|}{208} \\
\hline LGS Controlled Modes & 167.94 & & 187.14 & \\
\hline AO Architecture & & 129.91 & & 149.38 \\
\hline Telescope & & 39.11 & & 39.11 \\
\hline NFIRAOS & & 48.52 & & 61.1 \\
\hline Science Instrument & & 35.78 & & 35.78 \\
\hline LGSF & & 30 & & 30 \\
\hline AO Components & & 72.56 & & 72.56 \\
\hline NGS Controlled Modes & 67.53 & & 67.86 & \\
\hline AO Architecture & & 49.57 & & 50.02 \\
\hline Telescope & & 36.8 & & 36.8 \\
\hline NFIRAOS & & 22.36 & & 22.36 \\
\hline Science Instrument & & 15.78 & & 15.78 \\
\hline AO Components & & 0 & & 0 \\
\hline Margin & \multicolumn{2}{|c|}{47} & \multicolumn{2}{|c|}{60} \\
\hline
\end{tabular}

Table 3: LGS MCAO performance estimate breakdown for the "AO Architecture" (or fundamental error) terms

\begin{tabular}{|c|c|c|c|c|c|c|}
\hline \multirow{3}{*}{\begin{tabular}{|l|} 
\\
\end{tabular}} & \multirow{2}{*}{\multicolumn{3}{|c|}{$\frac{\text { On-Axis }}{\mathrm{CBE}}$}} & \multicolumn{3}{|c|}{ 34" x 34" FoV Average } \\
\hline & & & & \multicolumn{3}{|c|}{ CBE } \\
\hline & 130 & & & 149 & & \\
\hline LGS Control Loop & & 129 & & & 148 & \\
\hline DM fitting error & & & 74 & & & 74 \\
\hline DM projection error & & & 48 & & & 85 \\
\hline LGS WFS aliasing error & & & 26 & & & 26 \\
\hline Tomography Error & & & 48 & & & 53 \\
\hline TMT pupil Function & & & 14 & & & 4 \\
\hline Servo Lag & & & 18 & & & 17 \\
\hline LGS WFS non-linearity & & & 19 & & & 23 \\
\hline LGS WFS noise & & & 51 & & & 53 \\
\hline Simulation Undersampling & & & 48 & & & 48 \\
\hline Scintillation & & 18 & & & 18 & \\
\hline
\end{tabular}

\section{SUMMARY}

Significant progress has been accomplished in the design, prototyping and modeling of the TMT first light AO systems and AO components. Design and prototyping activities have taken place at the NRC-Herzberg Institute in Canada, at IOE in China, and at TMT in Pasadena to advance the NFIRAOS, LGSF and AOESW design effort. Progress in AO components development is also continuing for i) the final design and prototyping of the deformable mirrors, ii) the NFIRAOS WFS, with the fabrication and test of the full scale polar coordinate detector for the LGS WFS, and the design and prototyping of the LGS and NGS visible cameras, iii) the final design of the NFIRAOS RTC, iv) and the prototyping and testing of sodium guidestar lasers. Extensive work is also being performed in the area of AO system engineering and modeling to better define and consolidate our design and interface requirements.

\section{ACKNOWLEDGMENTS}

This paper is a summary of the contributions of numerous TMT staff members, TMT partners and TMT suppliers including: Luc Gilles, Angel Otárola, Tony Travouillon, Melissa Trubey, Lianqi Wang (TMT AO Group), Sean Adkins (Consultant), Hugh Thompson, Gelys Trancho (TMT), David R. Andersen, Jenny Atwood, Peter Byrnes, Kris Caputa, Jennifer Dunn, Joeleff Fitzsimmons, Glen Herriot, Alexis Hill, Dan Kerley, Malcolm Smith, Jean-Pierre Véran (NRCHerzberg), Kai Wei, Muwen Fan, Changchun Jiang, Min Li, Xiqi Li, Daoman Riu, Jinlong Tang, (IOE), Terry Bruno, Jeff Cavaco, Audrey Brooks (AOA Xinetics), Hubert Pagès, Jean-Christophe Sinquin, Aurelien Moreau, Gabrielle 
Dutey, Stephane Vaillant (CILAS), Bob Leach (ARC), Kyle Laudner (Quartus), Mark Fraser (M3 Engineering), James Gregory, Bradley Felton, Kevin Ryu, Michael Brattain, Daniel O’Mara (MIT/LL), Bo Yong, Junwei Zuo (TIPC).

The TMT Project gratefully acknowledges the support of the TMT collaborating institutions. They are the Association of Canadian Universities for Research in Astronomy (ACURA), the California Institute of Technology, the University of California, the National Astronomical Observatory of Japan, the National Astronomical Observatories of China and their consortium partners, and the Department of Science and Technology of India and their supported institutes. This work was supported as well by the Gordon and Betty Moore Foundation, the Canada Foundation for Innovation, the Ontario Ministry of Research and Innovation, the National Research Council of Canada, the Natural Sciences and Engineering Research Council of Canada, the British Columbia Knowledge Development Fund, the Association of Universities for Research in Astronomy (AURA), the U.S. National Science Foundation, and the National Institutes of Natural Sciences of Japan.

\section{REFERENCES}

[1] Liu F., "Thirty Meter Telescope project status," In Ground-based and Airborne Telescope VI Proc. of SPIE Vol. 9906, (2016).

[2] Simard L., "Thrity Meter science instruments: a status report", In Ground-based and Airborne Instrumentation for Astronomy VI Proc. of SPIE Vol. 9908, (2016).

[3] Wright, S. A., "The infrared imaging spectrograph (IRIS) for TMT: latest science cases and simulations," In Adaptive Optics Systems V Proc. of SPIE Vol. 9909, (2016).

[4] Herriot, G., et al., "NFIRAOS AO for the Thirty Meter Telescope," In Adaptive Optics Systems V Proc. of SPIE Vol. 9909, (2016).

[5] Véran, J.P., "Solving the NFIRAOS Calibration puzzle," In Adaptive Optics Systems V Proc. of SPIE Vol. 9909, (2016).

[6] Véran, J.P., et al., "Pyramid versus Shack-Hartmann: Trade Study Results for the NFIRAOS NGS WFS," AO4ELT4 Proceedings, (2015).

[7] Mieda, E., "Testing the Pyramid truth wavefront sensor for NFIRAOS in the lab," In Adaptive Optics Systems V Proc. of SPIE Vol. 9909, (2016).

[8] Fitzsimmons, J., "Design and analysis of the NFIRAOS thermal optics enclosure," In Ground-based and Airborne Instrumentation for Astronomy VI Proc. of SPIE Vol. 9908, (2016).

[9] Atwood, J., "NFIRAOS in 2015: engineering for future integration of complex sub-systems," In Adaptive Optics Systems V Proc. of SPIE Vol. 9909, (2016).

[10] Hill, A., "A prototype of the NFIRAOS to instrument thermo-mechanical interface," In Advances in Optical and Mechanical Technologies for Telescopes and Instrumentation Proc. of SPIE Vol. 9912, (2016).

[11]Lamontagne, F., "NFIRAOS beam splitters subsystems optomecahnical design," In Advances in Optical and Mechanical Technologies for Telescopes and Instrumentation Proc. of SPIE Vol. 9912, (2016).

[12] Rosensteiner, M., "On the verification of NFIRAOS algorithms and performance on the HeNOS bench," In Adaptive Optics Systems V Proc. of SPIE Vol. 9909, (2016).

[13] Turri, P., "Analysis and verification of the error budget for HeNOS: the test bench for NFIRAOS," In Adaptive Optics Systems V Proc. of SPIE Vol. 9909, (2016).

[14] Smith, M., "Thirty Meter Telescope narrow-field infrared adaptive optics system (NFIRAOS) real-time controller prototyping results," In Adaptive Optics Systems V Proc. of SPIE Vol. 9909, (2016).

[15] Kerley, D., "Thirty Meter Telescope narrow-field infrared adaptive optics system real-time controller preliminary architecture," In Software and Cyberinfrastructure for Astronomy IV Proc. of SPIE Vol. 9913, (2016).

[16] Li, M., "The progress of the TMT Laser Guide Star Facility," In Adaptive Optics Systems V Proc. of SPIE Vol. 9909, (2016).

[17] Pagès, H., "Developments of piezo deformable mirrors," In Adaptive Optics Systems V Proc. of SPIE Vol. 9909, (2016).

[18] Adkins, S., M., "Measured performance of the prototype polar coordinate CCD array," Adaptive Optics System III Proc. SPIE 8447, 0R1-16 (2012)

[19] Adkins, S. M., "Review of the latest developments in fast low noise detectors for wave-front sensing in the visible," Adaptive Optics Systems IV Proc. SPIE 9148, (2014). 
[20] Adkins, S. M., "Design and development of the full-scale polar coordinate detector", In Adaptive Optics Systems V Proc. of SPIE Vol. 9915, (2016).

[21] Enderlein, M., et al., "On-sky results of the TOPTICA guide star laser," In Adaptive Optics Systems V Proc. of SPIE Vol. 9909, (2016).

[22] Bonaccini, D., "LGS Return Flux: report on the Tenerife optimization experiments and comparison with the Toptica laser results at the VILT", In Adaptive Optics Systems V Proc. of SPIE Vol. 9909, (2016).

[23] Chin, J. C., "Keck II laser guide star AO system and performance with the Toptica/MPBC laser", In Adaptive Optics Systems V Proc. of SPIE Vol. 9909, (2016).

[24] Schöeck, M., "Flowdown of the TMT astrometry error budget(s) to the design of the first light instrument IRIS," In Ground-based and Airborne Instrumentation for Astronomy VI Proc. of SPIE Vol. 9908, (2016).

[25] Wang, L., "Optimizing LGS AO Performance in the context of evolving turbulence and sodium profiles," AO4ELT4 Proceedings, (2015). 